\title{
Ad hoc Editors
}

For serving as ad hoc editors of manuscripts published in 2006 we thank:

Helena Freitas

Susana Rodríguez

\section{Reviewers}

We thank the following colleagues who served as reviewers of manuscripts published in 2006:

Godfrey C. Akani

Mariajo Bañuelos

Keryn Bromberg

Ragan M. Callaway

Jordi Cortina

Emiliano Donadio

Enrique Figueroa

Nele Ingerpuu

József Lanszki

Francisco Lloret

Luca Luiselli

Cezary Mitrus

Ryan O'Donnell

Juan Manuel Pleguezuelos

Paula Sobral

Francisco Valera

Martin Zobel 